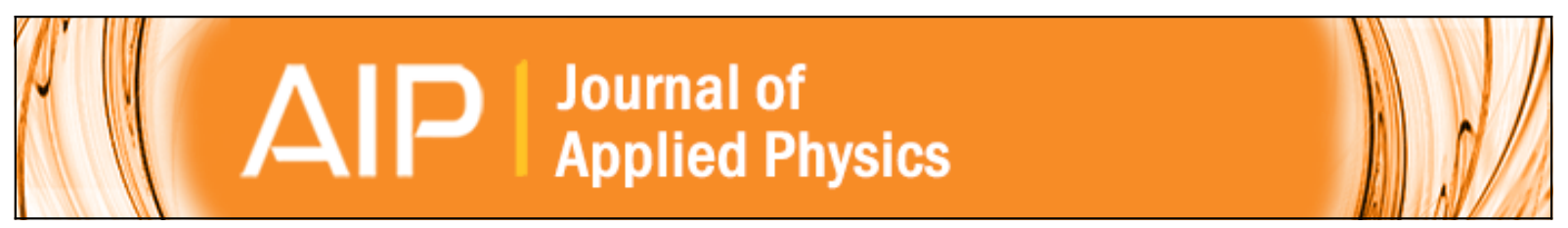

\title{
Strength of polycrystalline niobium from high-pressure x-ray diffraction data: A comparison of results from line-width and line-shift analyses
}

Anil K. Singh and Hanns-Peter Liermann

Citation: Journal of Applied Physics 118, 065903 (2015); doi: 10.1063/1.4927727

View online: http://dx.doi.org/10.1063/1.4927727

View Table of Contents: http://scitation.aip.org/content/aip/journal/jap/118/6?ver=pdfcov

Published by the AIP Publishing

\section{Articles you may be interested in}

High-pressure behavior and thermoelastic properties of niobium studied by in situ x-ray diffraction

J. Appl. Phys. 116, 013516 (2014); 10.1063/1.4887436

Strength and elasticity of niobium under high pressure

J. Appl. Phys. 109, 113539 (2011); 10.1063/1.3594748

Strength of polycrystalline coarse-grained platinum to $330 \mathrm{GPa}$ and of nanocrystalline platinum to $70 \mathrm{GPa}$ from high-pressure $x$-ray diffraction data

J. Appl. Phys. 103, 063524 (2008); 10.1063/1.2891424

Aluminum as a pressure-transmitting medium cum pressure standard for X-ray diffraction experiments to 200 GPa with diamond anvil cells

J. Appl. Phys. 101, 123526 (2007); 10.1063/1.2734868

High-pressure structure and equation of state study of nitrosonium nitrate from synchrotron x-ray diffraction J. Chem. Phys. 118, 8350 (2003); 10.1063/1.1566939

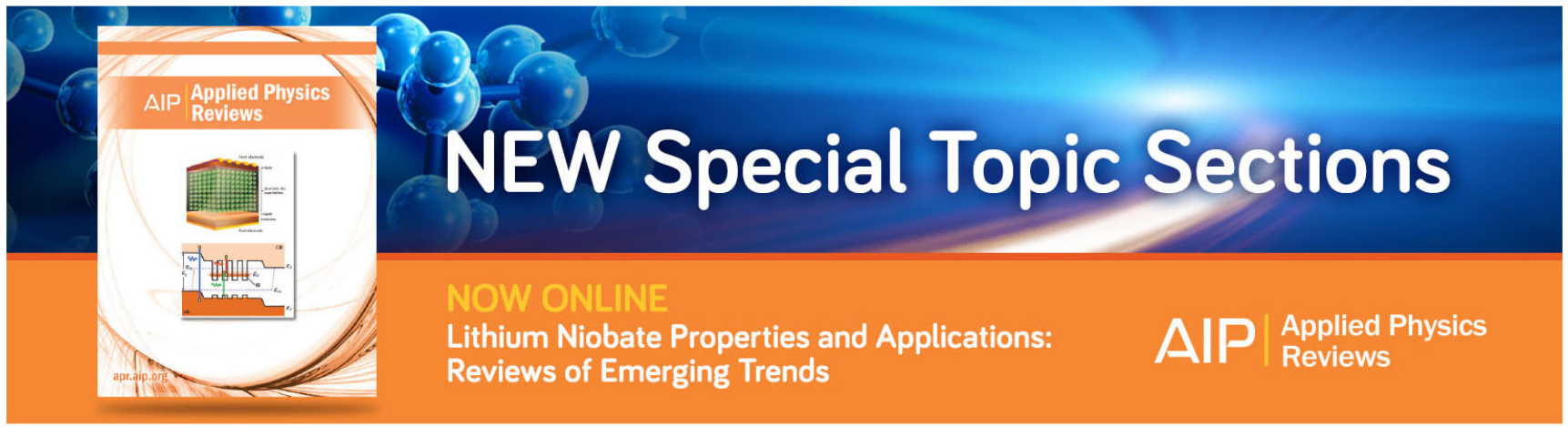




\title{
Strength of polycrystalline niobium from high-pressure x-ray diffraction data: A comparison of results from line-width and line-shift analyses
}

\author{
Anil K. Singh ${ }^{1, a)}$ and Hanns-Peter Liermann ${ }^{2}$ \\ ${ }^{1}$ Materials Science Division, Council of Scientific and Industrial Research (CSIR), \\ National Aerospace Laboratories, Bangalore 560 017, India \\ ${ }^{2}$ Photon Science, Deutsches Elektronen Synchrotron, Notkestr. 85, 22607, Hamburg, Germany
}

(Received 5 June 2015; accepted 21 July 2015; published online 12 August 2015)

\begin{abstract}
High purity polycrystalline niobium was compressed in a diamond anvil cell (DAC) without any pressure transmitting medium and the pressure was increased in steps of $\sim 5 \mathrm{GPa}$ to the highest pressure of $\sim 40 \mathrm{GPa}$. The diffraction pattern was recorded after each pressure increment using angle-dispersive mode with the conventional diffraction geometry, wherein the primary x-ray beam is parallel to the load axis of the DAC. The strength of niobium as function of pressure was determined using the line-width and line-shift analyses. Both $e Y$ and $2 e Y$, where $Y$ is the aggregate Young's modulus and $e$ is the strain determined from the line-width analysis, have been used as the measure of strength in earlier studies. In this study, it is $e Y$ that agrees with the strength determined from the line-shift analysis of the radial diffraction data as well as the data from the conventional diffraction geometry. These results have been discussed and compared with a similar observation made earlier on strength of diamond. This study highlights the ambiguity that presently exists in choosing $e Y$ or $2 e Y$ as a measure of strength while attempting to estimate the strength from the diffraction line width analysis. @ 2015 AIP Publishing LLC. [http://dx.doi.org/10.1063/1.4927727]
\end{abstract}

\section{INTRODUCTION}

A polycrystalline sample compressed in a diamond anvil cell (DAC) develops complex stresses and resulting strains. ${ }^{1}$ A simplified approach recognizes two types of strains: the micro- and macro-strains - the former results in the broadening of the diffraction lines and the latter in shift of the diffraction lines. The diffraction-line broadening has been used to determine the maximum stress in the stress distribution in the crystallites. ${ }^{2-12}$ The maximum stress $p_{\max }(\cong e Y$, where $e$ and $Y$ are the micro-strain and the aggregate Young's modulus, respectively) is often termed the micro-differential stress (micro-DS) and is taken as the measure of compressive strength of the polycrystalline sample material. Some studies used $p_{\max }$ (e.g., Refs. 2 and 5-7), whereas others $2 p_{\max }$ (e.g., Refs. 8-12) as the measure of micro-DS. The macro-stresses at the center of the sample compressed in a DAC possess a cylindrical symmetry about the load axis, the axial stress component $\sigma_{33}$ being larger than the radial stress component $\sigma_{11}$. The difference $\left(\sigma_{33}-\sigma_{11}\right)=t$, termed uniaxial stress or macro-DS, is another measure of strength at a confining pressure equaling the mean normal stress. ${ }^{13-18}$ Both the measures of strength, the micro- and macro-DS, are under identical confining pressure and must be equal. We determined strength of $\mathrm{Nb}$ to be $40 \mathrm{GPa}$ from the angle-dispersive $\mathrm{x}$-ray diffraction patterns taken with the conventional geometry of the DAC by using the methods outlined earlier for microDS $^{9}$ and macro-DS. ${ }^{17}$ These results have been compared with macro-DS obtained from the radial diffraction data in an earlier study. ${ }^{19}$

\footnotetext{
a) Author to whom correspondence should be addressed. Electronic mail: singhnal@yahoo.com
}

\section{THEORETICAL ANALYSIS}

\section{A. Line-width analysis}

The model for the microscopic stress distribution in the crystallites of the compressed aggregate assumes that all stresses between zero and a maximum stress $p_{\max }$ occur with equal probability. ${ }^{20}$ For a predominantly Gaussian profile, the apparent strain $\eta(h k l)$ perpendicular to the plane $(h k l)$ is given by ${ }^{3}$

$$
\left(2 w_{\mathrm{hkl}} \cos \theta_{\mathrm{hkl}}\right)^{2}=(\lambda / D)^{2}+\eta^{2}(h k l) \sin ^{2} \theta_{\mathrm{hkl}} .
$$

Here, $\left(2 w_{\mathrm{hkl}}\right)$ and $\theta_{\mathrm{hkl}}$ denote full width at half maximum (FWHM) on $2 \theta$-scale and the Bragg angle of the reflection $(h k l) . \lambda$ is the wavelength of the $\mathrm{x}$-rays and $D$ is the average apparent size of the crystallites assumed independent of $(h k l)$. Equation (1) can be rewritten in terms singlecrystal Young's modulus $Y(h k l)$ as follows: ${ }^{9}$

$$
\left(2 w_{\mathrm{hk} l} \cos \theta_{\mathrm{hkl}}\right)^{2}=(\lambda / D)^{2}+\left[\frac{4 p_{\mathrm{max}}}{Y(h k l)}\right]^{2} \sin ^{2} \theta_{\mathrm{hkl}} .
$$

For the cubic system, ${ }^{21}$

$$
Y(h k l)=S_{11}-2\left(S_{11}-S_{12}-\frac{1}{2} S_{44}\right) \Gamma(h k l) .
$$

Here, $S_{i j}$ are the single-crystal elastic compliances, and

$$
\Gamma(h k l)=\left(h^{2} k^{2}+k^{2} l^{2}+l^{2} h^{2}\right) /\left(h^{2}+k^{2}+l^{2}\right)^{2} .
$$

Equation (2) suggests that the $\left(2 w_{\mathrm{hkl}} \cos \theta_{\mathrm{hkl}}\right)^{2}$ versus $\left[\sin \theta_{\mathrm{hkl}} / Y(h k l)\right]^{2}$ plot is a straight line and $p_{\max }$ in terms of the slope $m(1)$ is given by 


$$
p_{\max }=\sqrt{m}(1) / 4
$$

Often, the elastic compliances $S_{i j}$ are not known. In such cases, the following equation obtained by replacing $\eta_{\mathrm{hkl}}$ in Eq. (1) by the average value $\langle\eta\rangle$ can be used. This leads to the following relation:

$$
\left(2 w_{\mathrm{hkl}} \cos \theta_{\mathrm{hkl}}\right)^{2}=(\lambda / D)^{2}+\langle\eta\rangle^{2} \sin ^{2} \theta_{\mathrm{hkl}} \cdot
$$

The average apparent strain $\langle\eta\rangle, p_{\max }$, slope $m(2)$, and aggregate Young' modulus $Y$ are related by

$$
p_{\max }=\langle\eta\rangle Y / 4=Y \sqrt{m(2)} / 4 .
$$

The equivalent equations applicable to energy dispersive diffraction technique can be found in the literature. ${ }^{22}$

It is to be noted that the apparent strain $\eta$, the strain $e$ produced by $p_{\max }$, and the root mean square strain $\varepsilon$ are related by ${ }^{23}$

$$
\eta=4 e=2(2 \pi)^{1 / 2} \varepsilon
$$

The strain $e$ is denoted by $\varepsilon$ in Refs. 5-7. References 11 and 12 use the same symbol $\varepsilon$ to denote $2 e$. It is seen from Eq. (8) that $p_{\max }=e Y$.

\section{B. Line-shift analysis}

It has been shown that the lattice parameter $a_{m}(h k l)$ measured using the angle-dispersive conventional geometry of the DAC plotted as function $3\left(1-3 \sin ^{2} \theta\right) \Gamma(h k l)$, termed gamma plot, is a straight line with a slope $M_{1}$ and intercept $M_{0}$ on the ordinate. ${ }^{17}$ The parameter $t$ is given by

$$
t=-\frac{3 M_{1}}{\alpha M_{0}\left(S_{11}-S_{12}-S_{44} / 2\right)} .
$$

Here, $\alpha$ is a weight parameter discussed at length in an earlier article. $^{24}$

\section{EXPERIMENTAL DETAILS}

The experiments were performed using stainless steel gaskets with the following dimensions: thickness- $290 \mu \mathrm{m}$, thickness in the indented region- $35 \mu \mathrm{m}$, and central hole diameter $-85 \mu \mathrm{m}$. A piece measuring $\approx 80 \times 80 \mu \mathrm{m}^{2}$ was cut from the as-supplied $\mathrm{Nb}$ rod and placed in the central hole. The $\mathrm{Nb}$ samples from Alfa-Aldrich had trace metal impurities (in ppm) below 1000, the major ones being Ta-700, $\mathrm{Si}-180, \mathrm{Mg}-5$, and $\mathrm{Ca}-2$. No pressure medium was used to obtain nonhydrostatic stresses characteristic of $\mathrm{Nb}$. The primary beam (cross-section $15 \times 15 \mu \mathrm{m}^{2}$ and wavelength of $0.03678 \mathrm{~nm}$ ) from HPCAT beam line was used. The pressure was increased in steps of $\sim 5 \mathrm{GPa}$ and the diffraction pattern recorded at each pressure on a Mar345 image plate.

The diffraction images were integrated using fit $2 \mathrm{~d}$ program $^{25}$ to give intensity versus $2 \theta$ diffraction profile (Fig. 1). The FWHM and $2 \theta_{0}$ of each diffraction line were determined by fitting a four-parameter pseudo-Voigt function to the diffraction data using the PeakFit program. To assess the diffraction-data quality and fit agreement of the diffraction

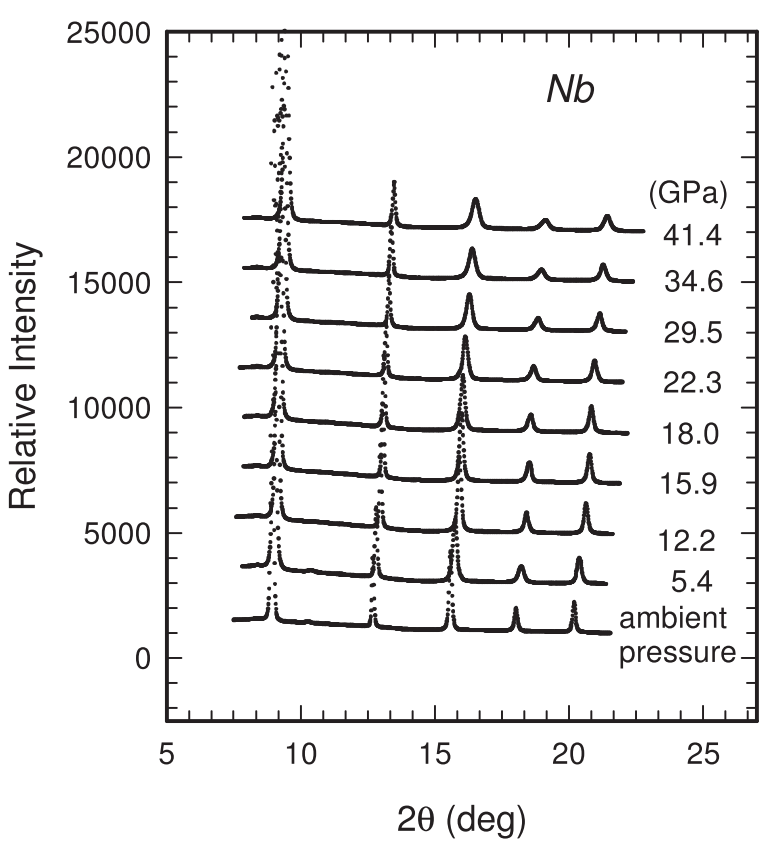

FIG. 1. The diffraction patterns of $\mathrm{Nb}$ from ambient pressure to $41.4 \mathrm{GPa}$ as recorded with conventional diffraction geometry of the DAC.

peaks, the patterns at ambient pressure and at $41.4 \mathrm{GPa}$ were selected. First five peaks with adequate background were separated (Figs. 2 and 3). A function of the type given below was fitted to each peak

$$
I=I_{0}[\eta L(2 \theta)+(1-\eta) G(2 \theta)]+2 \theta m+n,
$$

where

$$
L(2 \theta)=\left[1+\left(2 \theta-2 \theta_{0}\right)^{2} / w^{2}\right],
$$

and

$$
G(2 \theta)=\exp \left[-(\ln 2)\left(2 \theta-2 \theta_{0}\right)^{2} / w^{2}\right] .
$$

$L(2 \theta)$ and $G(2 \theta)$ are the Cauchy and Gaussian functions, respectively. ${ }^{26}$ The terms $I_{0}, 2 \theta_{0}, 2 w$, and $\eta$ are the peak intensity, peak position, FWHM, and weight parameter (shape factor), respectively. The last two terms in Eq. (10) represent linear background, the $m$ and $n$ being constants. The background-corrected peak-only profile agreement index ${ }^{27}$ $R_{p}^{\prime}$ was used to assess the goodness of fit. $R_{p}^{\prime}$ is defined by the relation

$$
R_{p}^{\prime}(\%)=\left[\sum_{i}\left|I_{i o}-I_{i c}\right| / \sum_{i}\left|I_{i o}-I_{i b}\right|\right] \times 100 .
$$

The parameters $I_{o}, I_{c}$, and $I_{b}$ are the observed, calculated from Eq. (10) with the fitted parameters, and the background intensities, respectively. The results of the fit are shown in Table I. The results obtained from the PeakFit program are also shown for comparison. Small values of $R_{p}^{\prime}$ indicate an excellent overall fit of Eq. (10) to the diffraction data. Further, as judged from the standard errors, the two parameters $2 \theta_{0}$ and $2 w$ that are used in the computation of $p_{\max }$ and $t$ are estimated with high degree of precision. It is seen from Table I that the individually fitted peak-parameters agree 

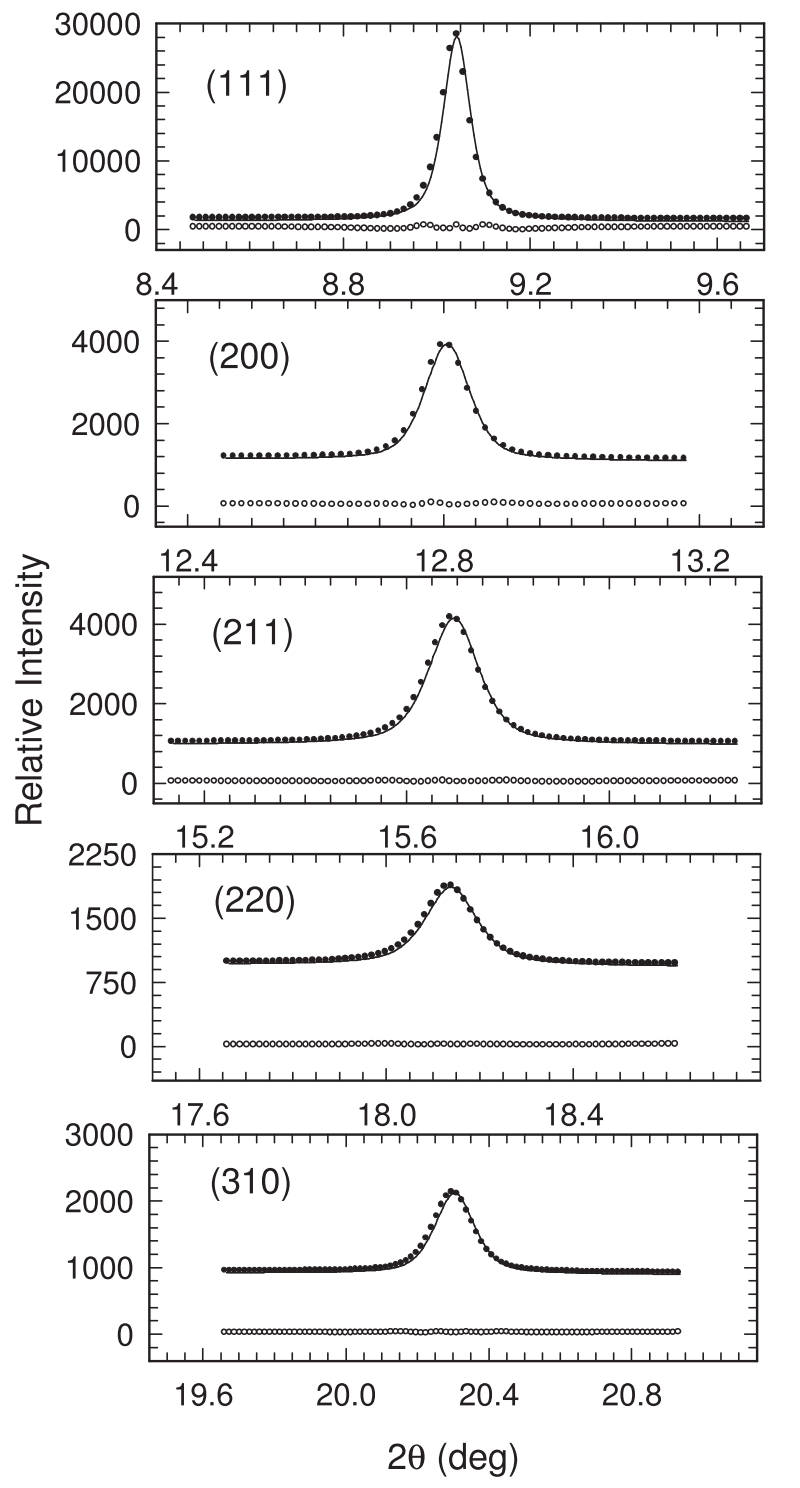

FIG. 2. The expanded views of intensity- $2 \theta$ profiles of the first five peaks (dots) recorded at ambient pressure. Continuous lines-fit of Eq. (10); unfilled circles- $\left(I_{o}-I_{c}\right)$ versus $2 \theta$ plots.

very well with the corresponding values obtained from the PeakFit program. Other patterns at the intermediate pressures give similar results.

\section{RESULTS AND DISCUSSION}

The ambient pressure single-crystal elastic moduli and their pressure derivatives needed to compute $Y(h k l)$ and $Y$ were taken from ultrasonic velocity measurement experiments. ${ }^{28}$ The elasticity data at high pressure were derived from a third order finite strain equation. ${ }^{29,30}$ It may be noted that the use of Eq. (9) requires the knowledge $\alpha_{0}$, the value of $\alpha$ relevant for $\mathrm{Nb}$. A three-parameter exponential decay function was fitted through the $\alpha_{0}$ versus pressure data taken from an earlier study. ${ }^{19}$ This function was used to compute the $\alpha_{0}$-value at any required pressure.

Typical results of fitting Eqs. (2) and (6) to the line width-data are shown in Figs. 4(a) and 4(b), respectively. It is noted that the scatter in Fig. 4(b) is much larger than that

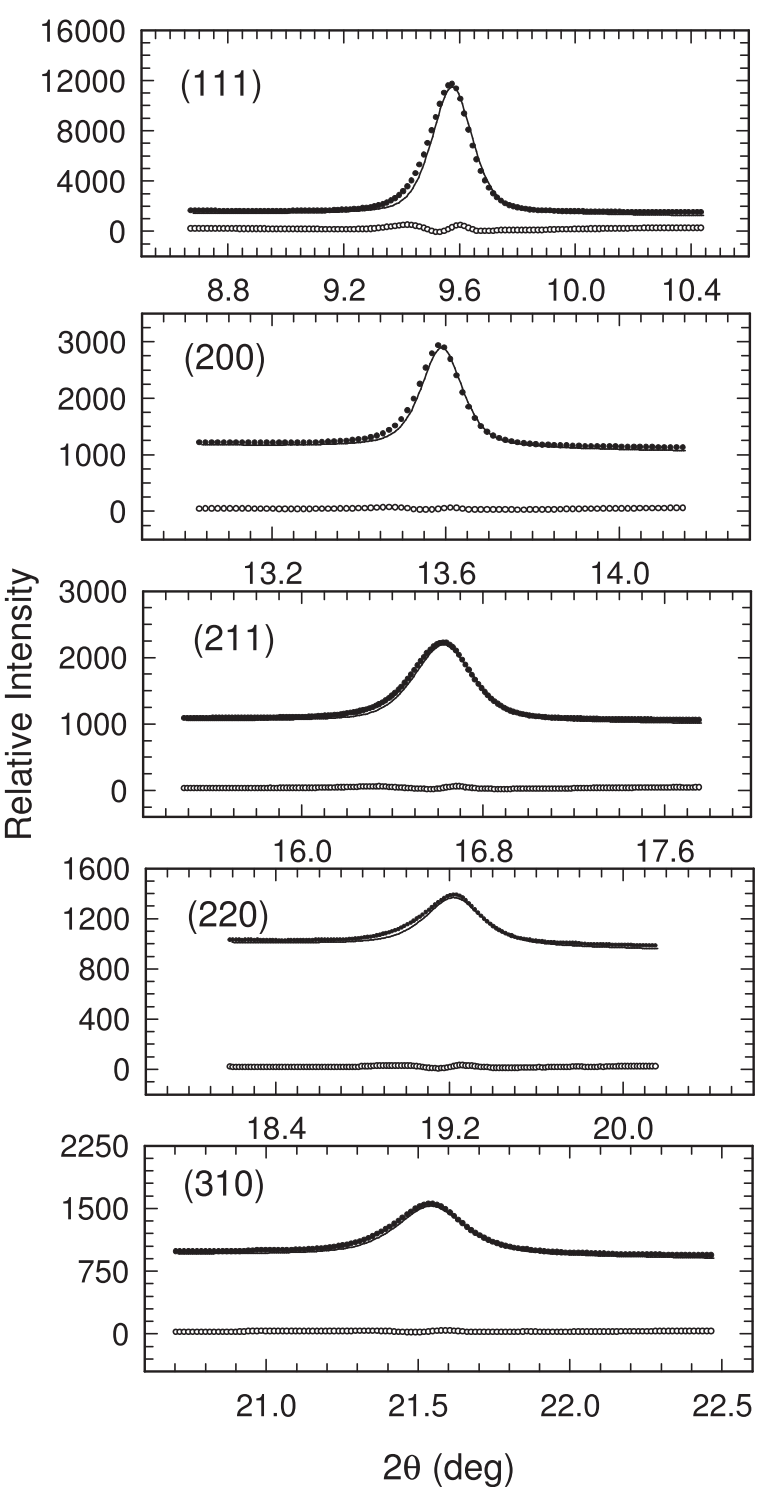

FIG. 3. The expanded views of intensity-2 $\theta$ profiles of the first five peaks recorded at $41.4 \mathrm{GPa}$. Symbols have the same denotation as in Fig. 2.

in Fig. 4(a). This is understandable as Eq. (6) ignores the $Y(h k l)$-dependence of $\operatorname{strain}^{20}$ that appears as the scatter in the fit. Fig. 5 shows typical gamma plots. The runs at pressures lower than $5.4 \mathrm{GPa}$ were discarded as the gamma plots for these runs were of poor quality. Fig. 6 shows the microDS derived from Eqs. (5) and (7) and $t$ (macro-DS) from Eq. (9). Also shown in Fig. 6 are $t$ versus pressure data obtained from the analysis of the radial diffraction patterns. ${ }^{19}$ It can be seen that $p_{\max }$ determined from Eq. (5) are marginally lower than those determined using Eq. (7). The $t-P$ data derived using Eq. (9) and the radial diffraction data ${ }^{19}$ agree well with $p_{\max }$.

Standard tensile tests at ambient pressure and temperature show that the yield stress of $\mathrm{Nb}$ is sensitive to the impurity levels. ${ }^{31}$ The samples with an impurity level of $1500 \mathrm{ppm}$ is $0.08 \mathrm{GPa}$ and increases to $0.21 \mathrm{GPa}$ for samples with impurity level of $6500 \mathrm{ppm}$. The estimated strength at ambient pressure from the present data is $0.2( \pm 0.1) \mathrm{GPa}$. Considering large errors in the estimated strength at ambient 
TABLE I. The parameters obtained by fitting Eq. (10) to the intensity-2 $\theta$ data at ambient pressure and $41.4 \mathrm{GPa}$. The standard error (S.E.) for each parameter is listed in the lower row. The numbers between the square brackets are obtained from the PeakFit program.

\begin{tabular}{|c|c|c|c|c|c|c|}
\hline & & 111 & 200 & 211 & 220 & 310 \\
\hline $\mathrm{I}_{0}$ & $0 \mathrm{GPa}$ & 26946 & 2819 & 3175 & 917 & 1209 \\
\hline S.E. & & 123 & 10 & 6 & 2 & 2 \\
\hline \multirow[t]{2}{*}{$2 w$} & & 0.0694 & 0.0834 & 0.1186 & 0.1316 & 0.1318 \\
\hline & & [0.069] & [0.083] & [0.119] & [0.132] & [0.131] \\
\hline \multirow[t]{2}{*}{ S.E. } & & 0.0004 & 0.0004 & 0.0004 & 0.0004 & 0.0002 \\
\hline & & {$[0.000]$} & {$[0.001]$} & {$[0.001]$} & [0.002] & [0.002] \\
\hline$\eta$ & & 0.65 & 0.47 & 0.57 & 0.65 & 0.50 \\
\hline S.E. & & 0.02 & 0.02 & 0.01 & 0.01 & 0.01 \\
\hline \multirow[t]{2}{*}{$2 \theta_{0}$} & & 9.0428 & 12.8056 & 15.6936 & 18.1388 & 20.3045 \\
\hline & & [9.0427] & [12.8055] & [15.6936] & [18.1387] & [20.3045] \\
\hline \multirow[t]{2}{*}{ S.E. } & & 0.0001 & 0.0001 & 0.0001 & 0.0001 & 0.0001 \\
\hline & & {$[0.0001]$} & {$[0.0001]$} & [0.0002] & [0.0002] & [0.0007] \\
\hline $\mathrm{m}$ & & -91.1 & -70.0 & -7.7 & -27.7 & -21.8 \\
\hline S.E. & & 39 & 9 & 3 & 1 & 1 \\
\hline $\mathrm{n}$ & & 2031 & 2010 & 1095 & 1451 & 1350 \\
\hline S.E. & & 350 & 113 & 52 & 25 & 19 \\
\hline $\mathrm{R}_{\mathrm{p}}^{\prime}(\%)$ & & 5.8 & 2.2 & 1.8 & 1.4 & 1.6 \\
\hline $\mathrm{I}_{0}$ & $41.1 \mathrm{GPa}$ & 10203 & 1785 & 1168 & 390 & 603 \\
\hline S.E. & & 57 & 7 & 4 & 2 & 2 \\
\hline \multirow[t]{2}{*}{$2 w$} & & 0.1656 & 0.1128 & 0.3104 & 0.3178 & 0.2734 \\
\hline & & [0.116] & [0.113] & [0.311] & [0.327] & [0.276] \\
\hline \multirow[t]{2}{*}{ S.E. } & & 0.0012 & 0.0006 & 0.0014 & 0.0026 & 0.0014 \\
\hline & & [0.001] & [0.001] & {$[0.002]$} & [0.007] & [0.005] \\
\hline$\eta$ & & 0.46 & 0.59 & 0.50 & 0.69 & 0.70 \\
\hline S.E. & & 0.03 & 0.02 & 0.02 & 0.03 & 0.02 \\
\hline \multirow[t]{2}{*}{$2 \theta_{0}$} & & 9.5746 & 13.5910 & 16.6262 & 19.2205 & 21.5431 \\
\hline & & 9.5745 & [13.5908] & [16.6268] & [19.2190] & [21.5419] \\
\hline \multirow[t]{2}{*}{ S.E. } & & 0.0004 & 0.0002 & 0.0005 & 0.0007 & 0.0004 \\
\hline & & [0.0003] & [0.0003] & $0.0007]$ & [0.0019] & {$[0.001]$} \\
\hline $\mathrm{m}$ & & -94 & -94 & -19 & -26 & -29 \\
\hline S.E. & & 19 & 4 & 1 & 1 & 1 \\
\hline $\mathrm{n}$ & & 2195 & 2395 & 1339 & 1475 & 1549 \\
\hline S.E. & & 182 & 52 & 22 & 16 & 20 \\
\hline $\mathrm{R}_{\mathrm{p}}^{\prime}(\%)$ & & 6.2 & 3.4 & 4.2 & 4.8 & 3.6 \\
\hline
\end{tabular}

pressure from high-pressure data, this estimate is in reasonable agreement with the ambient pressure strength data.

The strength increases steeply as the pressure is increased from the ambient, reaches a local maximum, and drops before it registers a monotonous increase beyond $15 \mathrm{GPa}$. This feature persists in the strength-pressure data derived from the line-width as well as the line-shift analyses. The pressure-dependence of strength in the 5-15 GPa region is anomalous. It may be noted that a similar trend in strength-pressure data was observed in an earlier study except that the minimum in the strength-pressure plot occurred at much higher pressure. ${ }^{17}$ The origin of this anomaly is not clear at present.

In a study on the strength of $\mathrm{MgO}$ by line width analysis, ${ }^{9} 2 p_{\max }$ was found to agree well with the strengthpressure data determined from the analysis of the radial diffraction data. ${ }^{32}$ The estimation of strength of $\mathrm{Fe}$ in the hexagonal phase ${ }^{33}$ showed that it was $2 p_{\max }$ that agreed with the strength derived from the radial diffraction data. ${ }^{34}$ These two

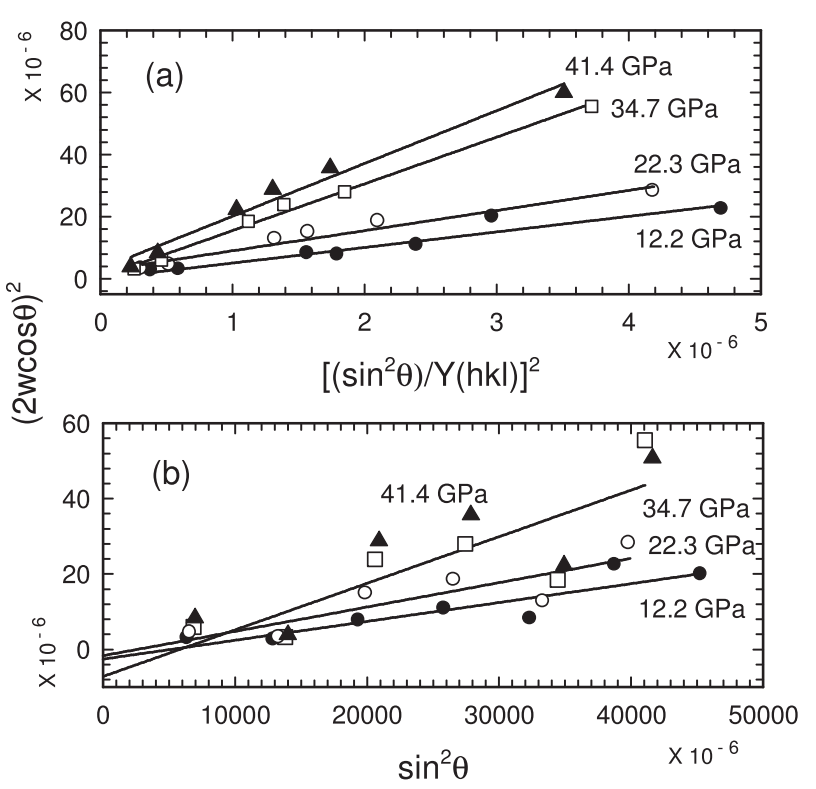

FIG. 4. Typical fits of (a) Eq. (2) and (b) Eq. (6) to the line-width (in arc radian) data. Because of large scatter, no line fitted through the data points at $41.4 \mathrm{GPa}$ in (b) is shown.

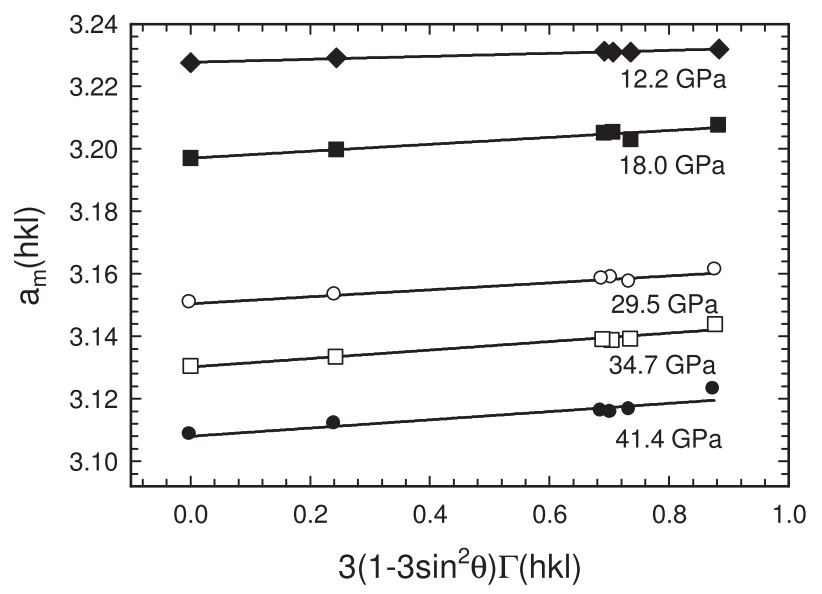

FIG. 5. The measured lattice parameter $a_{m}(h k l)$ versus $3\left(1-3 \sin ^{2} \theta\right) \Gamma(h k l)$ plots, termed gamma-plots, at different pressures.

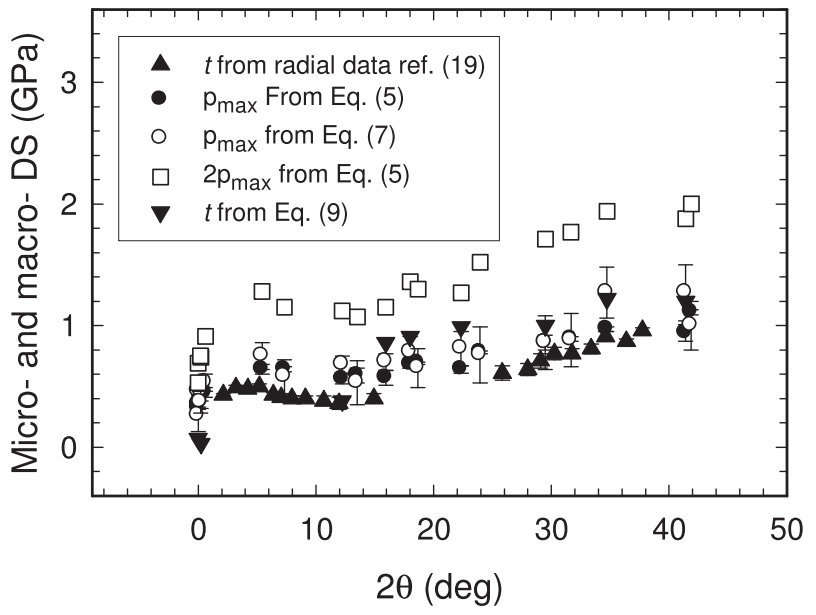

FIG. 6. Comparison of micro- and macro-differential stresses, $p_{\max }$ and $t$. 
examples probably led to a generalization that $2 p_{\max }$ is the correct measure of strength and would match the strength derived from the radial diffraction data. More recently, $2 p_{\max }$ was found to agree well with $t$ derived from the radial diffraction data on silica. ${ }^{35,36}$ Many other studies (e.g., Refs. 10-12) used $2 p_{\max }$ as the criterion of strength. In the present study, the use of $2 p_{\max }$ as the measure of strength will result in large disagreement with the macro-DS obtained either from Eq. (9) or from the radial diffraction data. ${ }^{19}$ Such differences between $2 p_{\max }$ and the macro-DS have been observed earlier in case of diamond. ${ }^{12}$ It was suggested $^{12}$ that this difference arose due to cupping of the diamond anvils at high pressure, which constrained the radial flow of the diamond sample and prevented $t$ from reaching the limiting value. However, this fails to explain the fact that the differences between $2 p_{\max }$ and macro-DS in case of both diamond and $\mathrm{Nb}$ persist down to the lowest pressures where the cupping of the anvil faces would tend to become insignificant. Another factor that could influence the estimates of strength from the radial diffraction data is the interference from the gasket that can prevent $t$ from reaching its maximum value. ${ }^{37}$ The gasket interference can become so dominant that it can result in negative $t$, as was observed in the compressibility studies of $\mathrm{Nb}^{38}$ It is interesting to note that in both cases, the present data and those on diamond, the use of $p_{\max }$ as the criterion of strength brings in a better agreement with the macro-DS. In case of $\mathrm{Nb}$, taking $2 p_{\max }$ as the measure of strength leads to another inconsistency in that the estimated strength at ambient pressure from $2 p_{\max }$ versus pressure data would reach $0.5 \mathrm{GPa}$, which is too large to match the strength obtained from the ambient pressure tensile tests. ${ }^{31}$ This supports the assumption that $p_{\max }$ represents the strength of $\mathrm{Nb}$.

\section{SUMMARY}

The strength versus pressure data of $\mathrm{Nb}$ derived from the line-shift data are found to be in good agreement with $p_{\text {max }}$. The ambient pressure strength estimated from the strength-pressure data is in good agreement with the yield strength determined from the standard tensile tests. This study highlights the ambiguity that exists in the criterion to be used while estimating the strength from the diffraction line-width analysis; many studies reported in the literature use $p_{\max }$ while others $2 p_{\max }$ as a measure of strength. Present understanding of the nature of micro-stresses in the sample and relation thereof with macro-DS does not appear to be adequate. Another area that lacks proper understanding is the role of the gasket in determining the deformation behavior of the sample.

\section{ACKNOWLEDGMENTS}

A.K.S. is a retired scientist from NAL.

${ }^{1}$ A. K. Singh, J. Phys. Chem. Solids 65, 1589 (2004).

${ }^{2}$ A. R. Stokes, K. J. Pascoe, and H. Lipson, Nature 151, 137 (1943).

${ }^{3}$ J. I. Langford, J. Appl. Crystallogr. 4, 164 (1971).

${ }^{4}$ D. J. Weidner, Y. Wang, and M. T. Vaughan, Geophys. Res. Lett. 21, 753, doi:10.1029/93GL03549 (1994).

${ }^{5}$ D. J. Weidner, Y. Wang, and M. T. Vaughan, Science 266, 419 (1994).

${ }^{6}$ J. Chen, Y. Yang, T. Yu, Z. Zhang, Y. Zhao, and L. Wang, High Pressure Res. 28, 423 (2008).

${ }^{7}$ J. Chen, T. Inoue, D. J. Weidner, Y. Wu, and M. T. Vaughan, Geophys. Res. Lett. 25, 575-578, doi:10.1029/98GL00043 (1998); Corrigendum 25, 1103, doi:10.1029/98GL00733 (1998).

${ }^{8}$ J. Chen, D. J. Weidner, and M. T. Vaughan, Nature (London) 419, 824 (2002).

${ }^{9}$ A. K. Singh, H. P. Liermann, and S. K. Saxena, Solid State Commun. 132, 795 (2004).

${ }^{10}$ A. K. Singh, H. P. Liermann, S. K. Saxena, H. K. Mao, and S. Usha Devi, J. Phys.: Condens. Matter 18, S969 (2006).

${ }^{11}$ D. He and T. S. Duffy, Phys. Rev. B 73, 134106 (2006).

${ }^{12}$ J. Wang, D. He, and T. S. Duffy, J. Appl. Phys. 108, 063521 (2010).

${ }^{13}$ A. L. Ruoff, J. Appl. Phys. 46, 1389 (1975).

${ }^{14}$ A. K. Singh, J. Appl. Phys. 73, 4278 (1993); Erratum 74, 5920 (1993).

${ }^{15}$ A. K. Singh, H. K. Mao, J. Shu, and R. J. Hemley, Phys. Rev. Lett. 80, 2157 (1998).

${ }^{16}$ A. K. Singh, C. Balasingh, H. K. Mao, R. J. Hemley, and J. Shu, J. Appl. Phys. 83, 7567 (1998).

${ }^{17}$ A. K. Singh and K. Takemura, J. Appl. Phys. 90, 3269 (2001); Erratum 91, 5507 (2002).

${ }^{18}$ A. K. Singh, J. Phys.: Conf. Ser. 500, 122005 (2014).

${ }^{19}$ A. K. Singh and H.-P. Liermann, J. Appl. Phys. 109, 113539 (2011).

${ }^{20}$ A. R. Stokes and A. J. C. Wilson, Proc. Phys. Soc. (London) 56, 174 (1944).

${ }^{21}$ J. F. Nye, Physical Properties of Crystals (Oxford University, London, 1960).

${ }^{22}$ L. Gerward, S. Morup, and H. Topsoe, J. Appl. Phys. 47, 822 (1976).

${ }^{23}$ H. P. Klug and L. E. Alexander, X-ray Diffraction Procedures for Polycrystalline and Amorphous Materials (Wiley, New York, 1973), pp. 661-662.

${ }^{24}$ A. K. Singh, J. Appl. Phys. 106, 043514 (2009).

${ }^{25}$ J. Hammersley, Fit2d user manual, ESRF, Grenoble, France, 1996.

${ }^{26}$ F. Sánchez-Bazo and F. L. Cumbrera, J. Appl. Crystallogr. 30, 427 (1997).

${ }^{27}$ R. J. Hill and R. X. Fischer, J. Appl. Crystallogr. 23, 462 (1990).

${ }^{28}$ K. W. Katahara, M. H. Manghanani, and E. S. Fisher, J. Phys. F: Met. Phys. 9, 773 (1979).

${ }^{29}$ F. Birch, J. Geophys. Res. 83(B3), 1257, doi:10.1029/JB083iB03p01257 (1978).

${ }^{30}$ A. K. Singh, H.-P. Liermann, Y. Akahama, S. K. Saxena, and E. Menéndez-Proupin, J. Appl. Phys. 103, 063524 (2008); see Eq. (7).

${ }^{31}$ L. I. Van Torne and G. Thomas, Acta Metall. 11, 881 (1963).

${ }^{32}$ S. Merkel, H. R. Wenk, J. Shu, G. Shen, P. Gillet, H.-K. Mao, and R. J. Hemley, J. Geophys. Res. 107(B11) 2271, doi:10.1029/2001JB000920 (2002).

${ }^{33}$ A. K. Singh, A. Jain, H. P. Liermann, and S. K. Saxena, J. Phys. Chem. Solids 67, 2197 (2006).

${ }^{34}$ S. Merkel, J. Shu, P. Gillet, H.-K. Mao, and R. J. Hemley, J. Geophys. Res. 110, B05201, doi:10.1029/2004JB003197 (2005).

${ }^{35}$ A. K. Singh, D. Andrault, and P. Bouvier, Phys. Earth Planet. Inter. 208-209, 1-10 (2012).

${ }^{36}$ S. R. Shieh, T. S. Duffy, and B. Li, Phys. Rev. Lett. 89, 255507 (2002).

${ }^{37}$ A. K. Singh, J. Phys.: Conf. Ser. 377, 012007 (2012).

${ }^{38}$ K. Takemura and A. K. Singh, Phys. Rev. B 73, 224119 (2006). 